\title{
Effects of continuous positive airway pressure therapy on left ventricular diastolic function: a randomised, sham- controlled clinical trial
}

\author{
Chi Young Shim ${ }^{1,4}$, Darae Kim ${ }^{1,4}$, Sungha Park ${ }^{1}$, Chan Joo Lee ${ }^{1}$, Hyung-Ju Cho ${ }^{2}$, \\ Jong-Won $\mathrm{Ha}^{1}$, Yang-Je $\mathrm{Cho}^{3}$ and Geu-Ru Hong ${ }^{1}$ \\ Affiliations: ${ }^{1}$ Division of Cardiology, Severance Cardiovascular Hospital, Seoul, Republic of Korea. ${ }^{2}$ Dept of \\ Otorhinolaryngology, Yonsei University College of Medicine, Seoul, Republic of Korea. ${ }^{3}$ Dept of Neurology, \\ Yonsei University College of Medicine, Seoul, Republic of Korea. ${ }^{4}$ Both authors contributed equally.
}

Correspondence: Geu-Ru Hong, Division of Cardiology, Severance Cardiovascular Hospital, Yonsei University College of Medicine, 50 Yonsei-ro, Seodaemun-gu, Seoul, Republic of Korea, 03722. E-mail: grhonglayuhs.ac

@ERSpublications

CPAP treatment for 3 months improved LV diastolic function more than sham treatment in patients with severe OSA http://ow.ly/kiYR30ggX5D

Cite this article as: Shim CY, Kim D, Park S, et al. Effects of continuous positive airway pressure therapy on left ventricular diastolic function: a randomised, sham-controlled clinical trial. Eur Respir J 2018; 51: 1701774 [https://doi.org/10.1183/13993003.01774-2017].

ABSTRACT Continuous positive airway pressure (CPAP) therapy may decrease left ventricular (LV) loads and improve myocardial oxygenation. In this study, we investigated the effect of CPAP on LV diastolic function compared with sham treatment in patients with severe obstructive sleep apnoea (OSA).

This 3-month prospective single-centre randomised sham-controlled trial analysed 52 patients with severe OSA. Patients were randomly assigned (1:1) to receive either CPAP or sham treatment for 3 months. The main investigator and patients were masked to the trial randomisation. The primary endpoint was change of early diastolic mitral annular $\left(\mathrm{e}^{\prime}\right)$ velocity over the 3-month period. Secondary end-points were pulse wave velocity (PWV), 24-h ambulatory blood pressure (BP) and variables of ventricular-vascular coupling at 3 months.

After 3 months of follow-up, CPAP treatment significantly increased the $\mathrm{e}^{\prime}$ velocity, and was greater than the sham treatment $\left(0.65 \pm 1.70\right.$ versus $\left.-0.61 \pm 1.85 \mathrm{~cm} \cdot \mathrm{s}^{-1}, \mathrm{p}=0.014\right)$. The PWV, 24-h mean diastolic $\mathrm{BP}$, night-time diastolic BP, arterial elastance index and ventricular-vascular coupling index after 3 months of follow-up decreased significantly in the CPAP group.

In patients with severe OSA, CPAP treatment for 3 months improved LV diastolic function more than sham treatment, and was accompanied by improvements in arterial stiffness and ventricular-vascular coupling.

This article has supplementary material available from erj.ersjournals.com

Received: Aug 302017 | Accepted after revision: Oct 302017

This study is registered at www.clinicaltrials.gov with identifier number NCT01854398.

Support statement: This study was supported by a Yuhan research grant and a faculty research grant at Yonsei University. Funding information for this article has been deposited with the Crossref Funder Registry.

Conflict of interest: None declared.

Copyright OERS 2018 


\section{Introduction}

Obstructive sleep apnoea (OSA) is a common chronic sleep disorder that is characterised by periodic reduction or cessation of breathing due to narrowing of the upper airways during sleep [1]. OSA is thought to be related to heart failure [2-4], as well as hypertension [5, 6], myocardial infarction [7] and arrhythmia [8], and exhibits episodic hypoxaemia, nocturnal sympathetic nervous system activation, elevated blood pressure (BP), and markers of oxidative stress, inflammation and hypercoagulability $[9,10]$.

Left ventricular (LV) diastolic dysfunction is often observed in patients with OSA, and is accompanied by common comorbidities, such as hypertension, obesity and diabetes, and consequent arterial stiffness and LV hypertrophy [11-14]. OSA contributes to the development of LV diastolic dysfunction and subsequent heart failure, even in patients with preserved ejection fraction $[3,4,11]$. Recent studies have suggested partial reversal of LV diastolic dysfunction after continuous positive airway pressure (CPAP) therapy through improved myocardial oxygenation, diminished LV pressure overload and sympathetic activation $[13,15,16]$. However, there were limited data proving the benefits of CPAP therapy on LV diastolic function from a randomised, sham-controlled clinical trial.

Therefore, in this study, we hypothesised that CPAP therapy for 3 months in patients with severe OSA would improve LV diastolic function when compared with sham treatment. In order to prove our hypothesis, we measured the effects of CPAP therapy on LV diastolic function in patients with severe OSA using comprehensive echocardiographic evaluations before and after 3 months of therapy. Moreover, we sought to characterise the underlying mechanisms involving LV diastolic dysfunction by a comprehensive assessment of arterial stiffness, BP variables and ventricular-vascular coupling.

\section{Methods}

\section{Study design and patients}

A prospective, single-centre, randomised, sham-controlled trial of parallel groups was conducted at Severance Hospital (Seoul, Republic of Korea). The trial was designed to determine the 3-month effects of CPAP on LV diastolic function in patients with severe OSA. This study was approved by the Severance Hospital institutional review board and registered with www.clinicaltrials.gov (NCT01854398). All patients provided informed consent.

60 patients with a confirmed diagnosis of severe OSA were enrolled. Severe OSA was defined as an apnoea/hypopnoea index (AHI) $>30$ events $\cdot h^{-1}$, as established by overnight polysomnography using the recommended hypopnoea criteria of the American Academy of Sleep Medicine (AASM) [17]. All participants were screened according to their history, clinical examination, baseline blood tests, 12-lead ECG and transthoracic echocardiography results. Exclusion criteria were LV ejection fraction $<50 \%$, prior history of coronary artery disease, arrhythmia, significant valvular dysfunction (of a moderate or greater degree), uncontrolled BP $(\geqslant 180 / 110 \mathrm{mmHg}$ ), chronic kidney disease (estimated glomerular filtration rate $<60 \mathrm{~mL} \cdot \mathrm{min}^{-1}$ ) or treatment with anxiolytics or sedatives. 56 eligible consenting patients underwent baseline BP measurements (peripheral BP, central BP and 24-h ambulatory BP monitoring), pulse wave velocity (PWV) determination and diastolic stress echocardiography.

\section{Randomisation and masking}

After baseline evaluation, patients who met eligibility criteria were randomly assigned (1:1) into parallel groups to receive either CPAP therapy $(n=28)$ or sham therapy $(n=28)$ for 3 months. Random assignment of patients was based on computer-generated random numbers unknown to the investigators. All patients were masked to the treatment. Thus, they were not aware whether they were receiving CPAP treatment or sham treatment. The investigators who assessed the end-points were unaware of the randomisation status of the patients, and did not set up or maintain the machines or assist the patients. The neurologist (Yang-Je Cho) who was assigned to monitor the treatment did not participate in end-point assessments. Therefore, despite the physical nature of the treatment, the study was effectively double blind.

\section{Procedures}

Patients were provided with a CPAP machine (S9 Elite with H5i; ResMed, San Diego, CA, USA). All study patients underwent standard CPAP titration to deliver pressures of $4-20 \mathrm{cmH}_{2} \mathrm{O}$ to eliminate obstructive events. CPAP titration was performed at the hospital as manual titration under full polysomnography, and patients received fixed pressures. Patients who were randomised to the sham interventions were fitted with one of two nasal mask interfaces (Mirage FX Nasal Mask or Swift FX Nasal Pillows Mask; ResMed) and scheduled for a full night of polysomnography using sham-CPAP. The sham system had modified masks which had an internal flow restricter and additional holes for effective washout of carbon dioxide. The sham system was designed to provide a patient experience similar to the true CPAP therapy. The pump-tubing-mask system with exhalation ports of sham equipment delivered similar sensations of flow 
from the mask and provided visual user interface feedback and operating sounds; however, the sham system was designed to deliver subtherapeutic CPAP of $<1 \mathrm{cmH}_{2} \mathrm{O}$.

Patients in the CPAP group started fixed-pressure CPAP at titrated pressure. Over a 3-month period, patients visited the neurologist on a monthly basis, and CPAP pressure was adjusted according to the results generated by the CPAP machine. CPAP compliance was monitored and/or recorded at patients' monthly visits throughout the study. CPAP was considered to be satisfactory when used minimally for $4 \mathrm{~h}$ per night for $\geqslant 6$ nights per week. 26 OSA patients completed this part of the study with a mean duration of CPAP therapy of 3 months before the second examination.

During the 3 months, two patients in each group discontinued interventions. All measurements were repeated after the 3-month study period. Ultimately, 52 patients were analysed for the end-points (figure 1). We checked that any drugs taken for hypertension had not been changed.

\section{Polysomnography}

Overnight polysomnography was performed using Embletta X100 system (Natus Embla Systems, Oakville, ON, Canada), a type-2 fully unattended portable system approved by the AASM. This type-2 portable monitor incorporates a minimum of seven channels, including two electroencephalography channels. This allowed accurate calculation of the AHI and was an improvement over the rough estimates that were obtained by type- 3 or -4 portable systems [18]. Manual laboratory CPAP titration was performed using in-laboratory polysomnography (REMStar Auto; Respironics, Pittsburgh, PA, USA and Comet XL system; Grass Technologies, West Warwick, RI, USA).

\section{Diastolic stress echocardiography}

Diastolic stress echocardiography with simultaneous respiratory gas analysis was performed using a variable load bicycle ergometer (Medical Positioning, Kansas City, MO, USA) with the subject in the supine position. Echocardiographic examination was performed simultaneously using ultrasound machines (Vivid 7 or Vivid E9 system; GE Healthcare, Chicago, IL, USA) with a $2.5-\mathrm{MHz}$ transducer as described

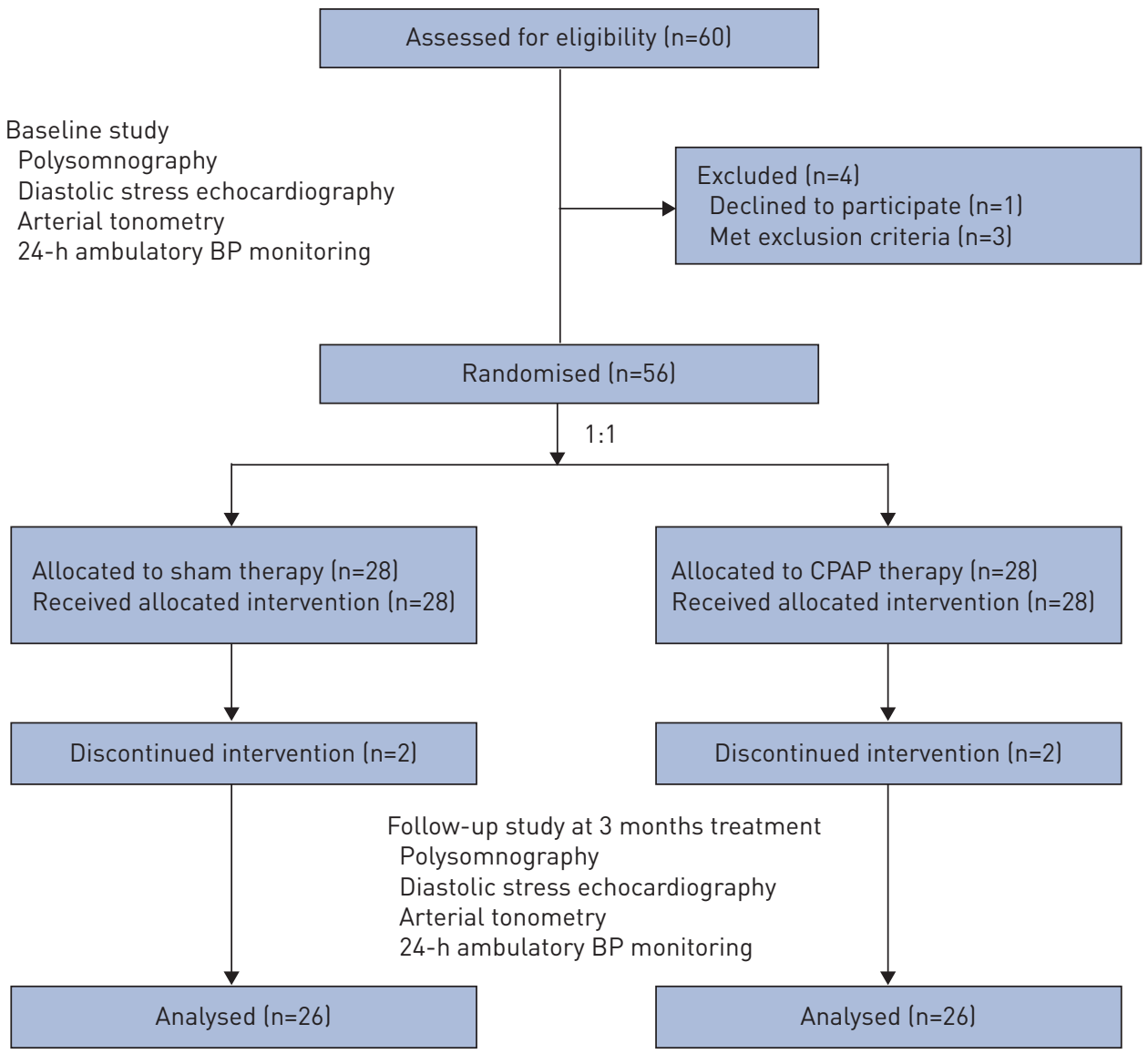

FIGURE 1 Trial profile. BP: blood pressure; CPAP: continuous positive airway pressure. 
previously $[19,20]$. From the resting images, standard two-dimensional and Doppler measurements were performed per the recommendations of the American Society of Echocardiography [21]. From the apical windows, a 1-2-mm pulse Doppler sample volume was placed at the mitral valve tip. The mitral inflow velocities were traced, and the following variables were obtained: peak velocity of early (E) and late (A) filling, and deceleration time of the E wave velocity. Stroke volume was measured from the LV outflow track diameter and pulse wave Doppler results. Early diastolic $\left(\mathrm{e}^{\prime}\right)$ and systolic $\left(\mathrm{s}^{\prime}\right)$ velocities of the mitral annulus were also measured on an apical four-chamber view with a 2-5-mm sample volume placed at the septal corner of the mitral annulus.

After obtaining standard rest images, multistage supine bicycle exercise testing was performed using a variable-load bicycle ergometer. The subjects pedalled at a constant speed beginning at a workload of $25 \mathrm{~W}$, with an incremental workload of $25 \mathrm{~W}$ every $3 \mathrm{~min}$ until limited by their symptoms. All measurements were performed at rest and at each stage of exercise in the same sequence. All data were digitally stored and analysed by two experienced sonographers who were blinded to the clinical and central haemodynamic data.

End-systolic pressure (ESP) was estimated as systolic $\mathrm{BP} \times 0.9$, as described previously [22]. Arterial elastance (Ea) was estimated as ESP/stroke volume. The arterial elastance index (EaI) was estimated by normalising the Ea to body surface area [23]. End-systolic ventricular elastance (Ees) was calculated by ESP/end-systolic volume [23]. Then, the ventricular elastance index (EesI) was calculated by normalising the Ees to body surface area. Finally, the ventricular-vascular coupling index was determined by the EaI/ EesI ratio. These measurements were performed at baseline, $25 \mathrm{~W}$ and $50 \mathrm{~W}$ of exercise in the same sequence.

\section{Arterial tonometry}

The radial pulse wave and PWV were assessed by arterial tonometry (SphygmoCor; AtCor Medical, Sydney, Australia). These measurements were collected in the supine position after a minimum rest of 5 min just before echocardiography. Central systolic BP, diastolic BP, pulse pressure (PP), augmentation pressure, augmentation indices, an index normalised for a heart rate of 75 bpm (AIx@75) were derived using pulse waveform analysis techniques. PP amplification was calculated as the ratio of peripheral to central PP. PWV was assessed at rest using the quotient of the carotid-femoral path length and carotid-femoral pressure pulse transit time. Pressure waveforms were gated with simultaneous electrocardiography and were used to calculate the PWV between two sites. Tonometry transit distances from the suprasternal notch to the radial, femoral and carotid recording sites were measured using a tape measure. The distances between recording sites were adjusted for parallel transmission in the aorta and the carotid sites by correcting for the distance between the suprasternal notch and the carotid site.

\section{The 24-h ambulatory BP monitoring}

Ambulatory BP monitoring was performed using an appropriately sized BP cuff on the nondominant arm, using a BP monitor (model 90207; SpaceLabs, Snoqualmie, WA, USA) programmed to record the BP every 30 min throughout the $24-\mathrm{h}$ period. Mean $24-\mathrm{h}$ BP was calculated as the mean of all the readings throughout the 24-h period. Night-time BP was defined as the BPs from the time the patient went to bed until the time the patient got out of bed, and daytime BP was defined as the BPs recorded during the rest of the day. Patients with $\pm 10 \%$ change in night-time BP from daytime BP were considered to be nondippers.

\section{Outcomes}

The primary end-point was the change of early diastolic mitral annular $\left(\mathrm{e}^{\prime}\right)$ velocity over the 3-month trial period. Secondary end-points were PWV, 24-h ambulatory BPs and variables of ventricular-vascular coupling at 3 months. In addition, we performed a subgroup analysis of treatment effects in patients according to the presence or absence of hypertension, baseline LV diastolic function and the severity of OSA (AHI $<50$ events $\cdot \mathrm{h}^{-1}$ or $\geqslant 50$ events $\cdot \mathrm{h}^{-1}$ ). Baseline diastolic function was classified into either preserved or impaired myocardial relaxation according to age-stratified normal reference of $\mathrm{e}^{\prime}$ velocity: $\mathrm{e}^{\prime}<10 \mathrm{~cm} \cdot \mathrm{s}^{-1}$ in patients aged $\leqslant 40$ years and $\mathrm{e}^{\prime}<7 \mathrm{~cm} \cdot \mathrm{s}^{-1}$ in patients aged $>40$ years were considered to represent impaired myocardial relaxation [24].

\section{Statistical analysis}

We chose a target sample of 60 patients to achieve $>46$ patients reaching the 3 -month assessment of the primary end-point. We calculated this sample size to provide $90 \%$ power with an $\alpha$-value of 0.05 . We expected a reduction of $20 \%$ in participants resulting from dropout because of withdrawals during the 3 months. A statistical analysis plan was completed before undertaking any data analyses. Group allocation 
was masked during the analysis of the primary outcome. The primary and secondary end-points were analysed in the per-protocol population.

Continuous variables are presented as mean $\pm \mathrm{SD}$ and categorical variables as a percentage of the group total. Differences between the two groups were analysed using two-sample t-tests. Paired comparisons before versus after CPAP or sham treatment were performed using paired t-tests. A p-value $<0.05$ was considered statistically significant. All analyses were performed in SPSS (version 22.0; IBM, Armonk, NY, USA).

\section{Results}

\section{Baseline characteristics}

Between May 1, 2013, and June 30, 2016, we recruited and screened patients for eligibility. 60 patients were eligible and we randomly assigned 28 to the sham treatment and 28 to the CPAP treatment. The per-protocol population was 26 patients in the sham therapy group and 26 patients in the CPAP therapy group. Their baseline characteristics are shown in table 1 . There were no differences between the two study groups, with the exception of higher body mass index and a higher prevalence of dyslipidaemia and statin usage in the CPAP group. The proportions of subject with hypertension in both groups were not different and the medication histories for BP control were comparable. Baseline polysomnography characteristics were not statistically different between the two groups.

\section{Adherence to the intervention}

Mean duration of use of the CPAP device did not differ between the two groups $\left(4.6 \pm 1.2 \mathrm{~h} \cdot \mathrm{day}^{-1}\right.$ for the active CPAP group and 5.0 $\pm 1.6 \mathrm{~h} \cdot \mathrm{day}^{-1}$ for the sham group, corresponding to 450 accumulated CPAP hours over 3 months for the CPAP group, and 414 accumulated CPAP hours over 3 months for the sham group). In the active CPAP group, the residual AHI during the 3 months of the trial intervention, measured by CPAP devices, averaged 3.5 events $\cdot h^{-1}$, indicating good control of OSA with active CPAP.

\section{Effects on LV diastolic function}

The effects of CPAP and sham treatment on LV structural or functional parameters are shown in table 2. LV structural variables including LV dimension, LV mass index and relative wall thickness were comparable between the two groups and were not changed significantly after the study period. LV ejection

\begin{tabular}{|c|c|c|c|}
\hline & Sham & CPAP & p-value \\
\hline Subjects & 26 & 26 & \\
\hline Age years & $48.8 \pm 10.7$ & $49.1 \pm 11.4$ & 0.920 \\
\hline Male & 24 (92) & 24 (92) & 1.000 \\
\hline Height $\mathrm{cm}$ & $169.9 \pm 7.3$ & $170.9 \pm 6.8$ & 0.583 \\
\hline Weight kg & $76.2 \pm 12.6$ & $81.7 \pm 11.7$ & 0.108 \\
\hline Body mass index $\mathrm{kg} \cdot \mathrm{m}^{-2}$ & $26.2 \pm 3.0$ & $27.8 \pm 2.8$ & 0.047 \\
\hline Body surface area $\mathrm{m}^{2}$ & $1.90 \pm 0.18$ & $1.96 \pm 0.18$ & 0.234 \\
\hline Hypertension & 15 (58) & $17(65)$ & 0.569 \\
\hline Diabetes mellitus & $1(4)$ & $4(15)$ & 0.158 \\
\hline Dyslipidaemia & $2(7)$ & $8(31)$ & 0.035 \\
\hline Current smokers & 6 (23) & 10 (39) & 0.229 \\
\hline \multicolumn{4}{|l|}{ Medications } \\
\hline Calcium channel blockers & $8(31)$ & $10(39)$ & 0.560 \\
\hline RAAS blockers & $8(31)$ & 9 (35) & 0.768 \\
\hline$\beta$-blockers & $4(15)$ & $4(15)$ & 1.000 \\
\hline Diuretics & $0(0)$ & $0(0)$ & 1.000 \\
\hline Statins & $2(7)$ & $8(31)$ & 0.035 \\
\hline Apnoea/hypopnoea index events $\cdot \mathrm{h}^{-1}$ & $53.4 \pm 20.5$ & $64.2 \pm 20.5$ & 0.063 \\
\hline Mean oxygen saturation $\%$ & $94.5 \pm 1.6$ & $93.9 \pm 1.7$ & 0.238 \\
\hline Sleep time with $\mathrm{SpO}_{2}$ saturation $<90 \% \%$ & $7.1 \pm 7.3$ & $10.5 \pm 11.6$ & 0.210 \\
\hline Average heart rate during sleep bpm & $62.3 \pm 6.3$ & $63.3 \pm 6.8$ & 0.576 \\
\hline Oxygen desaturation index events $\cdot \mathrm{h}^{-1}$ & $30.1 \pm 22.7$ & $27.5 \pm 21.6$ & 0.677 \\
\hline
\end{tabular}

Data are presented as $n$, mean \pm SD or $n(\%)$, unless otherwise stated. CPAP: continuous positive airway pressure; RAAS: renin-angiotensin-aldosterone system. $\mathrm{SpO}_{2}$ : arterial oxygen saturation measured by pulse oximetry. 
TABLE 2 Echocardiographic variables at baseline and 3 months after treatment

\begin{tabular}{|c|c|c|c|c|}
\hline & \multicolumn{2}{|c|}{ Sham } & \multicolumn{2}{|c|}{ CPAP } \\
\hline & Baseline & Follow-up & Baseline & Follow-up \\
\hline Subjects & \multicolumn{2}{|c|}{26} & \multicolumn{2}{|c|}{26} \\
\hline LVEDD mm & $49 \pm 3$ & $48 \pm 3$ & $50 \pm 3$ & $49 \pm 2$ \\
\hline LVESD mm & $32 \pm 3$ & $31 \pm 2$ & $32 \pm 3$ & $32 \pm 2$ \\
\hline LVEF \% & $67 \pm 5$ & $67 \pm 5$ & $69 \pm 5$ & $67 \pm 6$ \\
\hline Relative wall thickness & $0.38 \pm 0.05$ & $0.40 \pm 0.04$ & $0.40 \pm 0.04$ & $0.40 \pm 0.04$ \\
\hline $\mathrm{LV}$ mass index $\mathrm{g} \cdot \mathrm{m}^{-2}$ & $85.7 \pm 12.8$ & $83.9 \pm 14.1$ & $92.0 \pm 15.0$ & $91.2 \pm 11.9$ \\
\hline $\begin{array}{l}\text { LA volume index } \mathrm{mL} \cdot \mathrm{m}^{-2} \\
\mathrm{e}^{\prime} \text { velocity } \mathrm{cm} \cdot \mathrm{s}^{-1}\end{array}$ & \multicolumn{3}{|c|}{$\mathrm{e}^{\prime}$ velocity $\mathrm{cm} \cdot \mathrm{s}^{-1}$} & $26.6 \pm 5.2$ \\
\hline At rest & $9.4 \pm 2.7$ & $8.7 \pm 2.9$ & $7.7 \pm 2.2^{*}$ & $8.3 \pm 2.3$ \\
\hline At $25 \mathrm{~W}$ & $11.1 \pm 2.2$ & $10.6 \pm 2.1$ & $9.6 \pm 1.7^{*}$ & $9.9 \pm 2.1$ \\
\hline At $50 \mathrm{~W}$ & $12.0 \pm 2.4$ & $11.7 \pm 3.0$ & $10.6 \pm 2.1^{*}$ & $10.5 \pm 2.6$ \\
\hline \multicolumn{5}{|l|}{$E / e^{\prime}$} \\
\hline At rest & $7.6 \pm 1.5$ & $7.9 \pm 1.8$ & $8.9 \pm 2.4^{*}$ & $8.9 \pm 2.4$ \\
\hline At $25 \mathrm{~W}$ & $8.3 \pm 1.9$ & $8.3 \pm 1.8$ & $9.3 \pm 2.1$ & $9.3 \pm 2.7$ \\
\hline At $50 \mathrm{~W}$ & $8.7 \pm 2.1$ & $8.8 \pm 1.9$ & $9.7 \pm 2.4$ & $10.1 \pm 3.4$ \\
\hline \multicolumn{5}{|l|}{$\mathrm{s}^{\prime}$ velocity $\mathrm{cm} \cdot \mathrm{s}^{-1}$} \\
\hline At rest & $8.4 \pm 1.6$ & $9.1 \pm 2.0$ & $8.7 \pm 1.4$ & $8.9 \pm 1.6$ \\
\hline At $25 \mathrm{~W}$ & $9.0 \pm 1.3$ & $9.2 \pm 1.8$ & $9.0 \pm 1.5$ & $8.7 \pm 1.7$ \\
\hline At $50 \mathrm{~W}$ & $10.3 \pm 1.6$ & $10.2 \pm 1.9$ & $10.0 \pm 1.8$ & $9.8 \pm 1.3$ \\
\hline
\end{tabular}

Data are presented as $\mathrm{n}$ or mean \pm SD. CPAP: continuous positive airway pressure; LVEDD: left ventricular end-diastolic dimension; LVESD: left ventricular end-systolic dimension; LVEF: left ventricular ejection fraction; LV: left ventricle; LA: left atrium; e': early diastolic mitral annular tissue; E/e': ratio of mitral inflow early filling wave to myocardial early diastolic mitral annular velocity; $\mathrm{s}^{\prime}$ : systolic mitral annular tissue. *: $p<0.05$ (t-test), compared with corresponding sham group; no data were significant at $p<0.05$ (paired t-test) compared with the value at baseline within the group.

fraction and s' velocities, which were markers of LV systolic function, were in the normal ranges in both groups either at baseline or after follow-up. In terms of LV diastolic function, the baseline $\mathrm{e}^{\prime}$ velocity in the CPAP group $\left(7.7 \pm 2.2 \mathrm{~cm} \cdot \mathrm{s}^{-1}\right)$ was significantly lower than that in the sham group $\left(9.4 \pm 2.7 \mathrm{~cm} \cdot \mathrm{s}^{-1}\right.$; $\mathrm{p}=0.017$ ), suggesting a more impaired LV relaxation in the CPAP group. Similarly, baseline E/e', reflecting LV filling pressure, was significantly higher in the CPAP group than in the sham group. However, after the 3-month treatment, those values were similar in both groups. The 3-month CPAP treatment revealed a significant improvement of LV diastolic function compared with the sham treatment (change of $\mathrm{e}^{\prime}$ velocity $0.65 \pm 1.70$ versus $-0.61 \pm 1.85 \mathrm{~cm} \cdot \mathrm{s}^{-1} ; \mathrm{p}=0.014$ ) (figure $2 \mathrm{a}$ ).

\section{Effects on BP and arterial stiffness}

As shown in table 3, peripheral BPs, central BPs and PP amplification in the two groups were not different at baseline and follow-up. Moreover, the changes of each parameter after 3 months of treatment were not significant in both groups. The PWV in the CPAP group at follow-up decreased considerably $\left(7.2 \pm 1.0 \mathrm{~m} \cdot \mathrm{s}^{-1}\right)$ compared to either baseline values $\left(8.0 \pm 1.3 \mathrm{~m} \cdot \mathrm{s}^{-1} ; \mathrm{p}<0.001\right)$ or the corresponding values $\left(8.1 \pm 1.0 \mathrm{~m} \cdot \mathrm{s}^{-1} ; \mathrm{p}=0.004\right)$ in the sham-treated group (figure $\left.2 \mathrm{~b}\right)$. Therefore, the change of PWV during the 3 -month treatment was significantly different between the groups $\left(-0.05 \pm 1.11 \mathrm{~m} \cdot \mathrm{s}^{-1}\right.$ in the sham group versus $-0.80 \pm 1.08 \mathrm{~m} \cdot \mathrm{s}^{-1}$ in the CPAP group; $\mathrm{p}=0.023$ ).

In terms of 24-h ambulatory BPs, night-time diastolic BPs and mean diastolic BPs at the 3-month follow-up in the CPAP group were significantly lower than the baseline values before randomisation. However, these BP-lowering effects were not visible in the sham-treated group.

\section{Effects on ventricular-vascular coupling}

Table 4 and figure 3 display each component of ventricular-vascular coupling and their changes in both groups. Baseline values of end-systolic volume index, stroke volume index, ESP, EaI, EesI and EaI/EesI were similar in both groups. However, resting stroke volume index at the 3-month follow-up in the CPAP group $\left(40.1 \pm 7.7 \mathrm{~mL} \cdot \mathrm{m}^{-2}\right)$ was significantly higher compared with the corresponding value in the sham group $\left(35.7 \pm 5.7 \mathrm{~mL} \cdot \mathrm{m}^{-2} ; \mathrm{p}=0.026\right)$. In the CPAP group, EaI at rest and at $25 \mathrm{~W}$ and $50 \mathrm{~W}$ exercise decreased significantly after the 3-month treatment. Finally, the ventricular-vascular coupling index (EaI/ EesI) at rest decreased considerably in the CPAP group ( $p=0.044)$, but not in the sham group $(\mathrm{p}=0.375)$. 

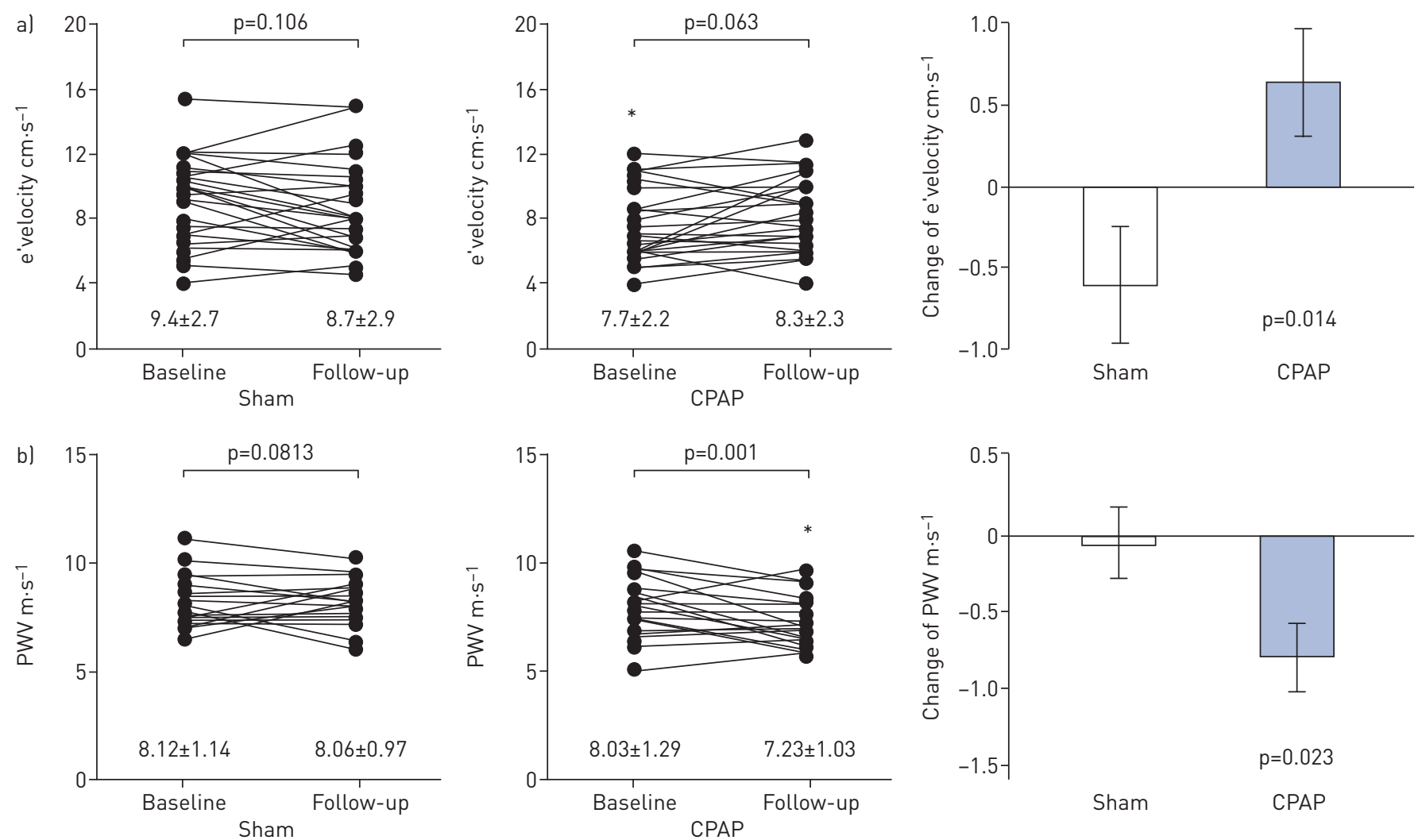

FIGURE 2 Changes of myocardial relaxation and arterial stiffness in the two study groups. a) Early diastolic mitral annular tissue (e') velocity; b) pulse wave velocity (PWV). CPAP: continuous positive airway pressure. *: $p<0.05$ by t-test, compared with the corresponding sham group.

Changes of LV diastolic function and arterial stiffness in the subgroups

Table 5 shows the results from subgroup analyses according to the presence of hypertension, baseline LV diastolic function and the severity of OSA. The results suggest that patients with hypertension and less severe OSA $\left(30 \leqslant \mathrm{AHI}<50\right.$ events $\left.\cdot \mathrm{h}^{-1}\right)$ would be more likely to benefit from CPAP therapy in improved LV diastolic function and arterial stiffness. Patients with both preserved and impaired myocardial relaxation showed significant benefits from CPAP therapy, compared to those in the sham group.

\section{Discussion}

The results of this trial indicate that 1) treatment with CPAP for 3 months in patients with severe OSA resulted in significantly improved LV diastolic function compared to sham treatment; 2) treatment with CPAP for 3 months in patients with severe OSA resulted in a significant improvement of arterial stiffness compared to sham treatment; and 3) significant enhancement of ventricular-vascular coupling was observed in the CPAP group and this result was associated with improved arterial elastance. To the best of our knowledge, the present study is the first to comprehensively demonstrate the effects of CPAP on LV diastolic function and the pathophysiological mechanisms in patients with severe OSA, using a randomised, sham-controlled clinical trial.

Observational studies have suggested that OSA contributes to the development of LV diastolic dysfunction and subsequent heart failure, even in patients with preserved ejection fraction $[3,4,11]$. Although several studies have suggested a role for OSA as an independent risk factor for the development of diastolic dysfunction, there have been inconsistent data, depending on the methodological approaches used to detect early diastolic dysfunction and the selection of the study populations $[13,25,26]$. In a study by KIM et al. [26], only patients with severe OSA showed a significant impairment of LV diastolic function as assessed by $\mathrm{e}^{\prime}$ velocity from tissue Doppler imaging, and LV diastolic dysfunction was associated with the severity of OSA independent of body mass index, diabetes mellitus and hypertension. Therefore, in the present study, we evaluated LV diastolic function using diastolic stress echocardiography as well as tissue Doppler imaging to detect early diastolic dysfunction and its changes after CPAP treatment in patients with severe OSA. 
TABLE 3 Peripheral, central and 24-h ambulatory blood pressure (BP) variables at baseline and 3 months after treatment

\begin{tabular}{|c|c|c|c|c|}
\hline & \multicolumn{2}{|c|}{ Sham } & \multicolumn{2}{|c|}{ CPAP } \\
\hline & Baseline & Follow-up & Baseline & Follow-up \\
\hline Subjects $\mathrm{n}$ & \multicolumn{2}{|c|}{26} & \multicolumn{2}{|c|}{26} \\
\hline \multicolumn{5}{|l|}{ Peripheral } \\
\hline Systolic BP mmHg & $137.2 \pm 16.6$ & $131.6 \pm 14.4$ & $135.2 \pm 14.9$ & $131.0 \pm 11.7$ \\
\hline Diastolic BP $\mathrm{mmHg}$ & $80.8 \pm 11.4$ & $78.7 \pm 10.5$ & $83.3 \pm 19.6$ & $78.1 \pm 9.8$ \\
\hline $\mathrm{PP} \mathrm{mmHg}$ & $56.4 \pm 11.6$ & $52.9 \pm 8.1$ & $57.0 \pm 13.8$ & $52.9 \pm 8.6$ \\
\hline \multicolumn{5}{|l|}{ Central } \\
\hline Systolic BP mmHg & $117.5 \pm 28.7$ & $115.5 \pm 11.6$ & $115.3 \pm 26.8$ & $115.9 \pm 9.8$ \\
\hline Diastolic BP mmHg & $82.4 \pm 11.8$ & $80.4 \pm 10.8$ & $82.8 \pm 12.3$ & $79.5 \pm 10.0$ \\
\hline $\mathrm{PP} \mathrm{mmHg}$ & $38.2 \pm 11.4$ & $35.2 \pm 4.4$ & $35.7 \pm 9.4$ & $36.4 \pm 8.2$ \\
\hline $\mathrm{AP} \mathrm{mmHg}$ & $7.2 \pm 6.0$ & $5.1 \pm 5.9$ & $5.4 \pm 5.4$ & $5.8 \pm 5.4$ \\
\hline Alxa75 \% & $18.8 \pm 27.0$ & $13.8 \pm 13.8$ & $15.8 \pm 18.8$ & $14.0 \pm 10.1$ \\
\hline PWV $\mathbf{m} \cdot \mathrm{s}^{-1}$ & $8.1 \pm 1.1$ & $8.1 \pm 1.0$ & $8.0 \pm 1.3$ & $7.2 \pm 1.0^{*, \#}$ \\
\hline PP amplification & $1.43 \pm 0.19$ & $1.51 \pm 0.23$ & $1.56 \pm 0.41$ & $1.48 \pm 0.20$ \\
\hline Heart rate bpm & $69.2 \pm 9.0$ & $72.6 \pm 11.1$ & $74.0 \pm 13.0$ & $72.7 \pm 10.4$ \\
\hline \multicolumn{5}{|l|}{ 24-h ambulatory BP } \\
\hline Mean systolic BP mmHg & $126.9 \pm 24.6$ & $123.4 \pm 10.9$ & $130.1 \pm 17.5$ & $125.4 \pm 12.3$ \\
\hline Mean diastolic BP mmHg & $82.9 \pm 14.7$ & $78.6 \pm 7.4$ & $83.7 \pm 13.9$ & $78.8 \pm 8.5^{*}$ \\
\hline Daytime systolic BP mmHg & $137.6 \pm 19.1$ & $130.2 \pm 11.7$ & $135.0 \pm 17.9$ & $131.8 \pm 13.0$ \\
\hline Daytime diastolic BP mmHg & $87.4 \pm 14.5$ & $82.2 \pm 6.9$ & $87.4 \pm 13.8$ & $83.1 \pm 9.1$ \\
\hline Night-time systolic BP mmHg & $118.0 \pm 20.7$ & $112.6 \pm 13.1$ & $120.8 \pm 17.3$ & $114.9 \pm 12.7$ \\
\hline Night-time diastolic BP $\mathrm{mmHg}$ & $74.9 \pm 15.2$ & $71.9 \pm 8.7$ & $77.0 \pm 13.8$ & $71.5 \pm 8.5^{*}$ \\
\hline Nondipper $\mathrm{n}(\%)$ & $5(20)$ & $9(36)$ & $5(20)$ & $8(31)$ \\
\hline \multicolumn{5}{|c|}{$\begin{array}{l}\text { Data are presented as mean } \pm \mathrm{SD} \text {, unless otherwise stated. CPAP: continuous positive airway pressure; PP: } \\
\text { pulse pressure; AP: augmentation pressure; AlxA75: augmentation index adjusted at heart rate of } 75 \mathrm{bpm} ; \\
\text { PWV: pulse wave velocity. }{ }^{*}: p<0.05 \text { (paired t-test), compared with the value at baseline within the group; } \\
{ }^{*}: \text { p }<0.05 \text { (t-test), compared with corresponding sham group. }\end{array}$} \\
\hline
\end{tabular}

CPAP is the standard therapy for patients with OSA. Recent studies have shown a partial reversal of LV diastolic dysfunction after CPAP therapy through improved myocardial oxygenation, diminished LV pressure overload and sympathetic activation [13, 15, 16]. A previous prospective randomised placebo-controlled crossover study reported that chronic application of nasal CPAP avoided the progression of diastolic abnormalities and might reverse these alterations, at least in the initial stages before the development of severe structural changes [13]. However, LV diastolic function was assessed by conventional Doppler parameters, such as the E/A ratio, deceleration time and isovolumic relaxation time, which are currently considered less sensitive and indirect measures of LV diastolic function than the $\mathrm{e}^{\prime}$ velocity obtained from tissue Doppler imaging. Therefore, in the present study, we evaluated LV diastolic function based on current clinical practice and guidelines, including tissue Doppler imaging techniques. Furthermore, changes in ventricular-vascular coupling were assessed from haemodynamic and volumetric assessment of the LV and systemic artery, because arterial stiffness and the resulting LV systolic and diastolic stiffness have been highlighted as key contributors to the pathophysiology of heart failure with preserved ejection fraction [27-29]. In addition, we have provided results regarding the improvement of LV diastolic function after CPAP treatment in comparison to sham treatment and have proposed a pathophysiological link between LV diastolic function and arterial stiffness through an improved LV diastolic function along with improved arterial stiffness, decreased night-time diastolic BP and decreased resting and exercise arterial elastance.

Previous studies reported that CPAP therapy promotes significant BP reduction $[30,31]$. In the current study, we found no significant improvement of peripheral BPs, central BPs and PP amplification. However, it should be noted that patients with uncontrolled BP were excluded from this study. In addition, almost half of the patients did not have hypertension, and the rest had already well-controlled hypertension at baseline. Such baseline characteristics may have precluded significant reduction of peripheral and central BPs. Interestingly, CPAP therapy significantly improved night-time diastolic BP and PWV compared to those in the sham group. We believe that effective CPAP therapy contributes to an increase in oxygenation and a reduction of sympathetic activity, as well as improvement of night-time BP. 
TABLE 4 Ventricular-vascular coupling at baseline and 12 weeks after randomisation

\begin{tabular}{|c|c|c|c|c|}
\hline & \multicolumn{2}{|c|}{ Sham } & \multicolumn{2}{|c|}{ CPAP } \\
\hline & Baseline & Follow-up & Baseline & Follow-up \\
\hline Subjects & \multicolumn{2}{|c|}{26} & \multicolumn{2}{|c|}{26} \\
\hline \multicolumn{5}{|l|}{ End-systolic volume index $\mathrm{mL} \cdot \mathrm{m}^{-2}$} \\
\hline At rest & $17.9 \pm 4.3$ & $16.3 \pm 4.2$ & $16.2 \pm 3.6$ & $16.0 \pm 3.2$ \\
\hline At $25 \mathrm{~W}$ & $18.5 \pm 4.0$ & $17.7 \pm 3.6$ & $16.8 \pm 4.0$ & $16.9 \pm 3.5$ \\
\hline At $50 \mathrm{~W}$ & $18.1 \pm 4.1$ & $16.9 \pm 3.4$ & $16.2 \pm 4.2$ & $15.9 \pm 3.4$ \\
\hline \multicolumn{5}{|l|}{ Stroke volume index $\mathrm{mL} \cdot \mathrm{m}^{-2}$} \\
\hline At rest & $36.0 \pm 4.8$ & $35.7 \pm 5.7$ & $37.2 \pm 9.1$ & $40.1 \pm 7.7^{\#}$ \\
\hline At $25 \mathrm{~W}$ & $39.9 \pm 4.6$ & $39.7 \pm 5.7$ & $40.5 \pm 8.7$ & $41.2 \pm 7.6$ \\
\hline At $50 \mathrm{~W}$ & $41.4 \pm 5.5$ & $41.7 \pm 6.1$ & $42.1 \pm 8.6$ & $43.5 \pm 7.1$ \\
\hline \multicolumn{5}{|l|}{ End-systolic pressure $\mathrm{mmHg}$} \\
\hline At rest & $115.0 \pm 14.4$ & $108.9 \pm 12.3$ & $113.4 \pm 17.6$ & $108.5 \pm 10.5$ \\
\hline At $25 \mathrm{~W}$ & $131.3 \pm 18.2$ & $118.7 \pm 12.8^{*}$ & $130.3 \pm 19.4$ & $118.8 \pm 12.3^{*}$ \\
\hline At $50 \mathrm{~W}$ & $136.2 \pm 19.3$ & $128.8 \pm 14.8^{*}$ & $138.2 \pm 20.2$ & $130.4 \pm 17.4$ \\
\hline \multicolumn{5}{|l|}{ Eal $\mathrm{mmHg} \cdot \mathrm{mL}^{-1} \cdot \mathrm{m}^{-2}$} \\
\hline At rest & $0.91 \pm 0.19$ & $0.89 \pm 0.24$ & $0.87 \pm 0.30$ & $0.75 \pm 0.22^{*, \#}$ \\
\hline At $25 \mathrm{~W}$ & $0.94 \pm 0.20$ & $0.85 \pm 0.19 *$ & $0.90 \pm 0.28$ & $0.80 \pm 0.25 *$ \\
\hline At $50 \mathrm{~W}$ & $0.95 \pm 0.25$ & $0.88 \pm 0.20$ & $0.92 \pm 0.32$ & $0.84 \pm 0.30 *$ \\
\hline \multicolumn{5}{|l|}{ Eesl $\mathrm{mmHg} \cdot \mathrm{mL}^{-1} \cdot \mathrm{m}^{-2}$} \\
\hline At rest & $1.94 \pm 0.62$ & $2.08 \pm 0.69$ & $1.98 \pm 0.70$ & $1.85 \pm 0.61$ \\
\hline At $25 \mathrm{~W}$ & $2.15 \pm 0.67$ & $2.07 \pm 0.74$ & $2.19 \pm 0.81$ & $1.93 \pm 0.71 *$ \\
\hline At $50 \mathrm{~W}$ & $2.30 \pm 0.70$ & $2.36 \pm 0.82$ & $2.47 \pm 1.02$ & $2.38 \pm 1.29$ \\
\hline \multicolumn{5}{|l|}{ Eal /Eesl } \\
\hline At rest & $0.50 \pm 0.13$ & $0.47 \pm 0.13$ & $0.46 \pm 0.12$ & $0.42 \pm 0.11 *$ \\
\hline At $25 \mathrm{~W}$ & $0.46 \pm 0.10$ & $0.44 \pm 0.10$ & $0.43 \pm 0.10$ & $0.43 \pm 0.11$ \\
\hline At $50 \mathrm{~W}$ & $0.44 \pm 0.11$ & $0.41 \pm 0.09$ & $0.40 \pm 0.13$ & $0.37 \pm 0.09$ \\
\hline \multicolumn{5}{|c|}{$\begin{array}{l}\text { Data are presented as } n \text { or mean } \pm \text { SD. CPAP: continuous positive airway pressure; Eal: arterial elastance } \\
\text { index; Eesl: ventricular elastance index; Eal/Eesl: ventricular-vascular coupling index. }{ }^{*}: p<0.05 \text { (paired } \\
\text { t-test), compared with the value at baseline within the group; }{ }^{\#}: p<0.05 \text { (t-test), compared with the } \\
\text { corresponding sham group. }\end{array}$} \\
\hline
\end{tabular}

Also of interest in this study was a significant increase in stroke volume after CPAP treatment compared with sham treatment, and this could be related to the pathophysiological effects of OSA on LV preload and afterload described in previous studies [2, 32, 33]. The combination of increased LV afterload, by surges in sympathetic nervous system activity and BP, and diminished LV preload, by leftward displacement of the interventricular septum at the time of exaggerated negative intrathoracic pressure against the occluded upper airway, have been reported to cause progressive reductions in stroke volume and cardiac output $[2,32,33]$. Moreover, it has been shown that treatment of OSA via CPAP largely reversed the overnight decreases in stroke volume and cardiac output [33], although the study was not a randomised, sham-controlled study. Likewise, one study demonstrated that CPAP treatment for 26 weeks in moderate to severe OSA with hypertension significantly improved ventricular-vascular coupling via a significant reduction in EaI [34]. However, the present study is the first to show the beneficial haemodynamic effects of CPAP therapy in severe OSA, compared with sham treatment, by a prospective, randomised clinical trial. A recent randomised trial without a sham control suggested that CPAP did not improve diastolic dysfunction in patients with coronary artery disease [35]. In our study, we excluded patients with coronary artery disease to avoid confounding comorbidities that affect diastolic function other than OSA. Additional sham-controlled randomised trials are needed to determine whether the benefit of CPAP therapy expands to OSA patients who are at high risk of cardiovascular problems.

The results from subgroup analyses provided important insights concerning the optimal therapeutic timing of CPAP in severe OSA regarding the aspects of reversing LV diastolic dysfunction. Despite the small number of patients, patients with AHI $<50$ events. $\mathrm{h}^{-1}$ showed a significant improvement of $\mathrm{e}^{\prime}$ velocity in the CPAP group compared with the sham group, but patients with AHI $\geqslant 50$ events.h ${ }^{-1}$ did not. These findings suggested that reversal of LV diastolic function after 3 months of CPAP therapy can be achieved if the severity of OSA is not extreme. In addition, patients with hypertension tended to experience more benefits from CPAP therapy. In summary, the results demonstrated that the pathophysiological mechanism relating to LV diastolic dysfunctions were linked to decreasing arterial stiffness. 

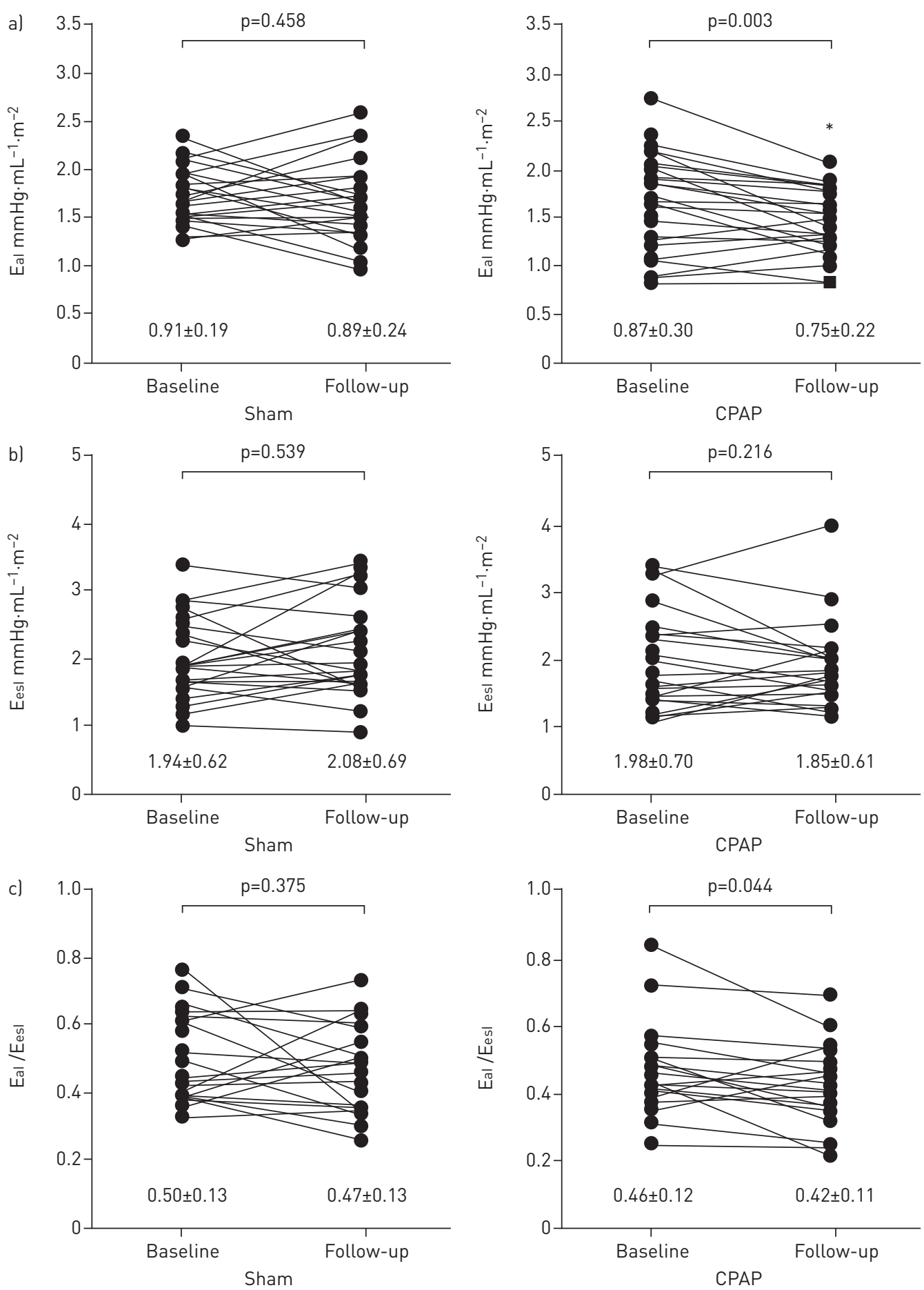

FIGURE 3 Changes of a) arterial elastance index (Eal), b) ventricular elastance index (Eesl) and c) ventricularvascular coupling index (Eal/Eesl) in the two study groups. CPAP: continuous positive airway pressure. *: $p<0.05$ by t-test, compared with the corresponding sham group.

Our study has some limitations. First, we excluded patients with reduced LV ejection fraction, a history of coronary artery disease, uncontrolled hypertension, significant valvular dysfunction and/or arrhythmia. Thus, our results should only be applied to populations that are similar to those eligible for our study. Second, despite randomisation, there were some between-group differences in baseline characteristics. The baseline $\mathrm{e}^{\prime}$ velocity in the CPAP group was lower than that in the sham-treated group. To adjust for the different baseline characteristics, the data were analysed in subgroups according to the baseline characteristics. In addition, regression analysis was performed in order to adjust differences in baseline 


\begin{tabular}{|c|c|c|c|}
\hline & Sham & CPAP & p-value \\
\hline \multicolumn{4}{|l|}{ Overall } \\
\hline Subjects & 26 & 26 & \\
\hline Change of $\mathrm{e}^{\prime}$ velocity & $-0.61 \pm 1.85$ & $0.65 \pm 1.70$ & 0.014 \\
\hline Change of PWV & $-0.05 \pm 1.11$ & $-0.80 \pm 1.08$ & 0.023 \\
\hline \multicolumn{4}{|l|}{ Without hypertension } \\
\hline Subjects & 11 & 9 & \\
\hline Change of $e^{\prime}$ velocity & $-0.14 \pm 0.71$ & $0.44 \pm 1.20$ & 0.401 \\
\hline Change of PWV & $0.33 \pm 1.33$ & $-0.56 \pm 1.21$ & 0.336 \\
\hline \multicolumn{4}{|l|}{ With hypertension } \\
\hline Subjects & 15 & 17 & \\
\hline Change of $\mathrm{e}^{\prime}$ velocity & $-0.95 \pm 1.93$ & $0.76 \pm 1.93$ & 0.018 \\
\hline Change of PWV & $-0.12 \pm 0.93$ & $-0.91 \pm 1.02$ & 0.041 \\
\hline \multicolumn{4}{|c|}{ Preserved myocardial relaxation } \\
\hline Subjects & 21 & 13 & \\
\hline Change of $e^{\prime}$ velocity & $-0.91 \pm 1.86$ & $0.24 \pm 0.89$ & 0.045 \\
\hline Change of PWV & $0.01 \pm 0.98$ & $-1.2 \pm 1.82$ & 0.023 \\
\hline \multicolumn{4}{|c|}{ Impaired myocardial relaxation } \\
\hline Subjects & 5 & 13 & \\
\hline Change of $\mathrm{e}^{\prime}$ velocity & $0.08 \pm 0.41$ & $1.61 \pm 1.51$ & 0.044 \\
\hline Change of PWV & $-0.28 \pm 1.62$ & $-0.80 \pm 1.01$ & 0.421 \\
\hline \multicolumn{4}{|c|}{ Apnoea/hypopnoea index $<50$ events $\cdot \mathrm{h}^{-1}$} \\
\hline Subjects & 12 & 8 & \\
\hline Change of $\mathrm{e}^{\prime}$ velocity & $-1.36 \pm 1.20$ & $0.68 \pm 2.04$ & 0.011 \\
\hline Change of PWV & $0.22 \pm 0.94$ & $-0.76 \pm 1.14$ & 0.056 \\
\hline \multicolumn{4}{|c|}{ Apnoea/hypopnoea index $\geqslant 50$ events $\cdot \mathrm{h}^{-1}$} \\
\hline Subjects & 14 & 18 & \\
\hline Change of $e^{\prime}$ velocity & $0.04 \pm 2.09$ & $0.64 \pm 1.58$ & 0.365 \\
\hline Change of PWV & $-0.28 \pm 1.22$ & $-0.81 \pm 1.08$ & 0.227 \\
\hline
\end{tabular}

Data are presented as $\mathrm{n}$ or mean $\pm \mathrm{SD}$, unless otherwise stated. CPAP: continuous positive airway pressure; $\mathrm{e}^{\prime}$ : early diastolic mitral annular tissue; PWV: pulse wave velocity.

characteristics; the change of $\mathrm{e}^{\prime}$ velocity was still significant in the active CPAP group compared to the sham group, even after adjustment of baseline characteristics (online supplementary table S5). Third, we included only two females in each group, so further studies are warranted in order to have confidence in our results for females with severe OSA. Last, some unidentified confounding factors might have affected the LV diastolic function during the 3-month treatment. However, we determined that cardiac medications and body weights did not change during the study period.

In conclusion, CPAP treatment for 3 months improved LV diastolic function more than sham treatment, and was accompanied by improvements in arterial stiffness and ventricular-vascular coupling in patients with severe OSA.

\section{Acknowledgements}

We thank the patients who participated in our trial and their relatives; ResMed for providing research equipment; and all physicians and research staff members for their hard work and enthusiasm.

Author contributions: G-R. Hong and Y-J. Cho designed this study after literature search. C.Y. Shim, S. Park, H-J. Cho and G-R. Hong contributed to the data collection. C.Y. Shim, D. Kim, Y-J. Cho and G-R. Hong contributed to the data analysis and interpretation. C.Y. Shim, D. Kim and C.J. Lee performed the statistical analysis of the primary end-points and reviewed other statistical analyses. C.Y. Shim and D. Kim wrote the first draft of the report. S. Park, H-J. Cho, J-W. Ha, Y-J. Cho and G-R. Hong reviewed and revised the manuscript critically for important intellectual content before submission. All authors approved the final submitted version.

\section{References}

1 Jordan AS, McSharry DG, Malhotra A. Adult obstructive sleep apnoea. Lancet 2014; 383: 736-747.

2 Kasai T, Bradley TD. Obstructive sleep apnea and heart failure: pathophysiologic and therapeutic implications. J Am Coll Cardiol 2011; 57: 119-127.

3 Mentz RJ, Kelly JP, von Lueder TG, et al. Noncardiac comorbidities in heart failure with reduced versus preserved ejection fraction. J Am Coll Cardiol 2014; 64: 2281-2293.

4 Arikawa T, Toyoda S, Haruyama A, et al. Impact of obstructive sleep apnoea on heart failure with preserved ejection fraction. Heart Lung Circ 2016; 25: 435-441. 
5 Peppard PE, Young T, Palta M, et al. Prospective study of the association between sleep-disordered breathing and hypertension. N Engl J Med 2000; 342: 1378-1384.

6 Nieto FJ, Young TB, Lind BK, et al. Association of sleep-disordered breathing, sleep apnea, and hypertension in a large community-based study. JAMA 2000; 283: 1829-1836.

7 Hung J, Witford EG, Parsons RW, et al. Association of sleep apnoea with myocardial infarction in men. Lancet 1990; 336: 261-264.

8 Guilleminault C, Connolly SJ, Winkle RA. Cardiac arrhythmia and conduction disturbances during sleep in 400 patients with sleep apnea syndrome. Am J Cardiol 1983; 52: 490-494.

9 McNicholas WT, Bonsigore MR. Sleep apnoea as an independent risk factor for cardiovascular disease: current evidence, basic mechanisms and research priorities. Eur Respir J 2007; 29: 156-178.

10 Phillips CL, McEwen BJ, Morel-Kopp MC, et al. Effects of continuous positive airway pressure on coagulability in obstructive sleep apnoea: a randomised, placebo-controlled crossover study. Thorax 2012; 67: 639-644.

11 Fung JW, Li TS, Choy DK, et al. Severe obstructive sleep apnea is associated with left ventricular diastolic dysfunction. Chest 2002; 121: 422-429.

12 Jelic S, Bartels MN, Matelika JH, et al. Arterial stiffness increases during obstructive sleep apneas. Sleep 2002; 25: 850-855.

13 Arias MA, García-Río F, Alonso-Fernández A, et al. Obstructive sleep apnea syndrome affects left ventricular diastolic function: effects of nasal continuous positive airway pressure in men. Circulation 2005; 112: 375-383.

14 Bodez D, Damy T, Soulat-Dufour L, et al. Consequences of obstructive sleep apnoea syndrome on left ventricular geometry and diastolic function. Arch Cardiovasc Dis 2016; 109: 494-503.

15 Akar Bayram N, Ciftci B, Durmaz T, et al. Effects of continuous positive airway pressure therapy on left ventricular function assessed by tissue Doppler imaging in patients with obstructive sleep apnoea syndrome. Eur J Echocardiogr 2009; 10: 376-382.

16 Butt M, Dwivedi G, Shantsila A, et al. Left ventricular systolic and diastolic function in obstructive sleep apnea: impact of continuous positive airway pressure therapy. Circ Heart Fail 2012; 5: 226-233.

17 Berry RB, Budhiraja R, Gottlieb DJ, et al. Rules for scoring respiratory events in sleep: update of the 2007 AASM Manual for the Scoring of Sleep and Associated Events. Deliberations of the Sleep Apnea Definitions Task Force of the American Academy of Sleep Medicine. J Clin Sleep Med 2012; 8: 597-619.

18 Flemons WW, Littner MR, Rowley JA, et al. Home diagnosis of sleep apnea: a systemic review of the literature. An evidence review cosponsored by the American Academy of Sleep Medicine, the American College of Chest Physicians, and the American Thoracic Society. Chest 2003; 124: 1543-1579.

19 Ha JW, Oh JK, Pellikka PA, et al. Diastolic stress echocardiography: a novel noninvasive diagnostic test for diastolic dysfunction using supine bicycle exercise Doppler echocardiography. J Am Soc Echocardiogr 2005; 18: 63-68.

20 Lancellotti P, Pellikka PA, Budts W, et al. The clinical use of stress echocardiography in non-ischaemic heart disease: recommendations from the European Association of Cardiovascular Imaging and the American Society of Echocardiography. Eur Heart J Cardiovasc Imaging 2016; 17: 1191-1229.

21 Lang RM, Bierig M, Devereux RB, et al. Recommendations for chamber quantification: a report from the American Society of Echocardiography's Guidelines and Standards Committee and the Chamber Quantification Writing Group, developed in conjunction with the European Association of Echocardiography, a branch of the European Society of Cardiology. J Am Soc Echocardiogr 2005; 18: 1440-1463.

22 Kelly RP, Ting CT, Yang TM, et al. Effective arterial elastance as index of arterial vascular load in humans. Circulation 1992; 86: 513-521.

23 Shim CY, Park S, Choi EY, et al. The relationship between ventricular-vascular uncoupling during exercise and impaired left ventricular longitudinal functional reserve in hypertensive patients. J Am Soc Hypertens 2013; 7: $198-205$.

24 Nagueh SF, Appleton CP, Gillebert TC, et al. Recommendations for the evaluation of left ventricular diastolic function by echocardiography. Eur J Echocardiogr 2009; 10: 165-193.

25 Niroumand M, Kuperstein R, Sasson Z, et al. Impact of obstructive sleep apnea on left ventricular mass and diastolic function. Am J Respir Crit Care Med 2001; 163: 1632-1636.

26 Kim SH, Cho GY, Shin C, et al. Impact of obstructive sleep apnea on left ventricular diastolic function. Am J Cardiol 2008; 101: 1663-1668

27 Kawaguchi M, Hay I, Fetics B, et al. Combined ventricular systolic and arterial stiffening in patients with heart failure and preserved ejection fraction: implications for systolic and diastolic reserve limitations. Circulation 2003; 107: 714-720.

28 Tan YT, Wenzelburger F, Lee E, et al. The pathophysiology of heart failure with normal ejection fraction: exercise echocardiography reveals complex abnormalities of both systolic and diastolic ventricular function involving torsion, untwist, and longitudinal motion. J Am Coll Cardiol 2009; 54: 36-46.

29 Shim CY, Park S, Choi D, et al. Sex differences in central hemodynamics and their relationship to left ventricular diastolic function. J Am Coll Cardiol 2011; 57: 1226-1233.

30 Schein AS, Kerkhoff AC, Coronel CC, et al. Continuous positive airway pressure reduces blood pressure in patients with obstructive sleep apnea; a systematic review and meta-analysis with 1000 patients. J Hypertens 2014; 32: $1762-1773$

31 Bratton DJ, Gaisl T, Wons AM, et al. CPAP vs mandibular advancement devices and blood pressure in patients with obstructive sleep apnea: a systematic review and meta-analysis. JAMA 2015; 314: 2280-2293.

32 Yumino D, Kasai T, Kimmerly D, et al. Differing effects of obstructive and central sleep apneas on stroke volume in patients with heart failure. Am J Respir Crit Care Med 2013; 187: 433-438.

33 Kasai T, Yumino D, Redolfi S, et al. Overnight effects of obstructive sleep apnea and its treatment on stroke volume in patients with heart failure. Can J Cardiol 2015; 31: 832-838.

34 Shantsila A, Santisila E, Butt M, et al. Ventricular-arterial coupling in obstructive sleep apnea. J Am Soc Hypertens 2014; 8: 624-629.

35 Glantz H, Johansson MC, Thunström E, et al. Effect of CPAP on diastolic function in coronary artery disease patients with nonsleepy obstructive sleep apnea: a randomized controlled trial. Int J Cardiol 2017; 241: 12-18. 